\title{
Phase plane analysis of walking with applications in controlling bipeds and prostheses
}

\begin{abstract}
In the Human Machine Integration laboratory, we have been designing wearable robots. For our robotic systems, the user's walking pattern and intent must be determined to generate appropriate motor outputs. In the past, we have used tunable gait patterns, variable stiffness patterns, and a tibia based controller. In this paper, we focus on understanding human gait and the underlying patterns by studying phase curves. For example, we use the progression of the shank phase angle as a metronome to determine the user's percent gait cycle. We go one step further by studying the phase angle of the shank as a function of the phase angle of the foot. A signature pattern between the two phase angles is shown. The pattern seems to be invariant to different tasks such as walking, walking quickly, and walking on slopes. In the future, it is hoped that a shank phase angle with a unique orientation and angular speed can be used to determine a unique foot phase angle allowing the generation of appropriate motor outputs.
\end{abstract}

Keywords: orientation, analysis, progression, phase angles, vertical, elevation, analyze
Volume 6 Issue I - 2020

\author{
Thomas G Sugar,' Emily H Sinitski,' Jason \\ MWilken, ${ }^{2}$ Sangram Redkar,' Mathew A \\ Holgate $^{3}$ \\ 'Polytechnic campus, Arizona State University, USA \\ ${ }^{2}$ Director of the military performance laboratory at BAMC, \\ USA \\ ${ }^{3}$ Tempe campus, Arizona State University, USA
}

Correspondence: Sangram Redkar, the Polytechnic School, Arizona State University, Mesa, AZ - 85212, USA, Emailssredka@gmail.com

Received: January 10, 2020 | Published: February 28, 2020

\section{Introduction}

In the Human Machine Integration laboratory, we have been designing wearable robots, specifically robotic ankle foot orthoses, ${ }^{1,2}$ robotic knee orthoses, and robotic ankle prostheses. ${ }^{3,4}$ For all of these robotic systems, the user's walking pattern and intent must be determined to generate appropriate motor outputs. In the past, we have used tunable gait patterns, ${ }^{3}$ variable stiffness patterns, ${ }^{1,5}$ and a new tibia based controller. ${ }^{6}$ The tibia based controller is very promising because the phase-plane diagram is used to determine the percent gait cycle. It also eliminates time directly from the analysis because the progression of the phase angle or phase angles is used as a metronome. The more important question is how do these phase angles of the tibia and foot progress in time? Do they follow each other? Does one loop around in two periods while the other loops around in one period? Is the relationship between the phase angles invariant to walking speed and other types of tasks? In this work, we study only the joint angles of the ankle and knee as well as the elevation angles of the foot and shank (tibia). We study these angles in walking, walking quickly, and walking up or down slopes. We are studying the elevation angle of the thigh and other tasks such as jogging and running, but will not discuss those results here.

Elevation angles are measured against the vertical component (measured against gravity), see Figure1. We believe they are extremely valuable because they are a direct measure of the moment caused by gravity against the member (or limb). Research groups, Lacquaniti and others, ${ }^{7-15}$ have shown that the elevation angles follow a planar covariance law. Computer graphics groups at the University of Pennsylvania have used the elevation angles to simulate/model gait. ${ }^{16}$ Blaya and Herr have also measured the elevation angles in their powered ankle foot orthosis. ${ }^{17,18}$

In this paper, we will analyze the joint angles and the elevation angles of the lower limb to determine if they can be used as inputs to our robotic systems.

\section{Methods}

Four subjects were recruited to participate in a single data collection session at the Center for the Intrepid, Brooke Army Medical Center. All subjects signed an IRB approved informed consent document prior to participating. Each subject was fitted with a minimum of four retroreflective markers per limb segment. A 26 camera Motion Analysis Corporation motion capture system collected marker position data at a rate of $120 \mathrm{~Hz}$. Walking surface slopes was modified using an instrumented motorized platform. Each subject was asked to walk over level ground at medium and fast speed. Subjects were asked to walk up and down a 10 degree slope at their self-selected pace.

Marker position data were labeled and recorded using EvART software (Motion Analysis Corp.). Visual 3D software (C-Motion Inc.) was used to calculate joint and segment displacements using an Euler angle approach. Matlab was used to analyze the raw angle data from Visual 3D. In this work, we did not filter any of the data, and the angular velocity data was calculated by a finite difference method using the diff function in Matlab. To calculate the phase angle, atan 2 and unwrap functions were used in Matlab to determine an angle between 0 and 360 degrees.

The "ankle angle" was determined using shank and foot local coordinate system data. The neutral or 0 degree position is observed when the long axis of the foot is perpendicular to the shank segment. The knee joint angle is measured between the thigh and shank with 0 degrees in the neutral standing position. This angle is labeled as "knee angle." In the right diagram of Figure 1, the angles are measured clockwise and are thus negative.

The foot elevation angle is measured in world coordinates between the foot and the vertical with 0 degrees in the neutral standing position. This angle is labeled as "foot angle." The shank elevation angle is measured in world coordinates between the shank and the vertical with 0 degrees in the neutral standing position. This angle is labeled as 
"shank angle." In the left diagram of Figure 1, the angles are measured clockwise and are thus negative. In some research fields, the elevation angles are called segmental angles. The segmental angles are the angles of the limb calculated in world coordinates.

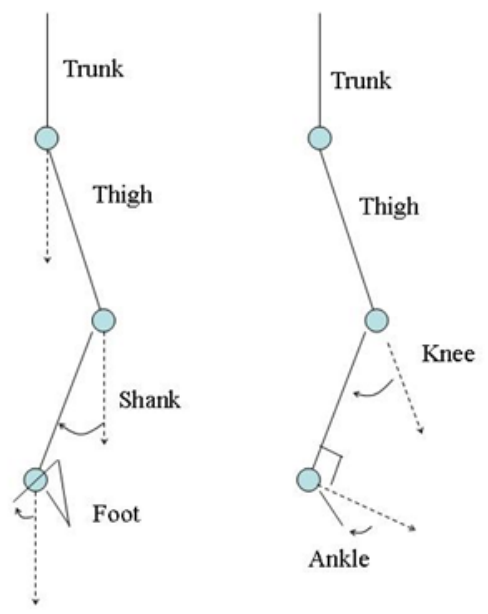

Figure I The definitions of the angles for the foot, shank, and knee are shown.

\section{Data analysis}

Ankle and foot angle data from six level walking cycles collected at medium speed are shown in Figure 2. The average gait cycle duration was 1.142 seconds. The foot elevation angle has one peak and one minimum per cycle. The ankle joint angle has two peaks and two minima per cycle. The elevation angle is very smooth and repeatable.

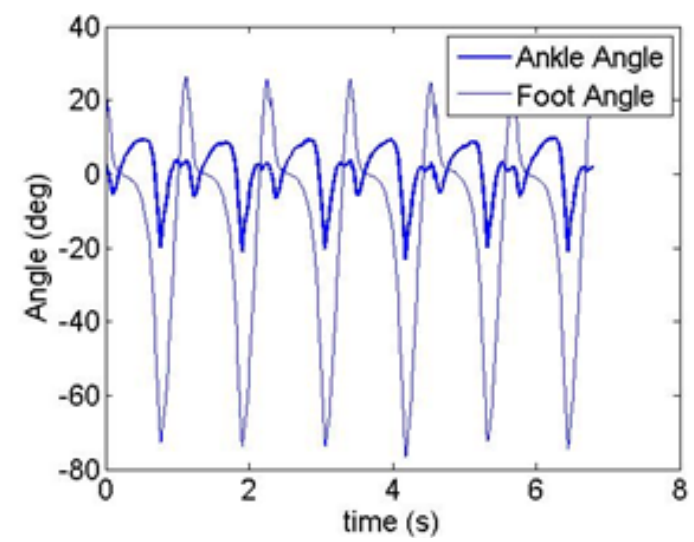

Figure $\mathbf{2}$ The ankle joint angle and foot elevation angle are shown for six gait cycles.

The ankle angle and foot angle were numerically differentiated to determine their respective angular velocities. A phase plot is shown in Figure 3 plotting the corresponding ankle angular velocity with the ankle angle at each point in the gait cycle. All six gait cycles are shown in Figure 3 with each phase plot making two loops per gait cycle.

A phase plot demonstrating the relationship between foot angular velocity and foot angle is shown in Figure 4. The data is smooth with little variability. Each of the six unique loops within the figure corresponds to a single gait cycle; therefore, each phase angle corresponds to a unique point in the gait cycle.

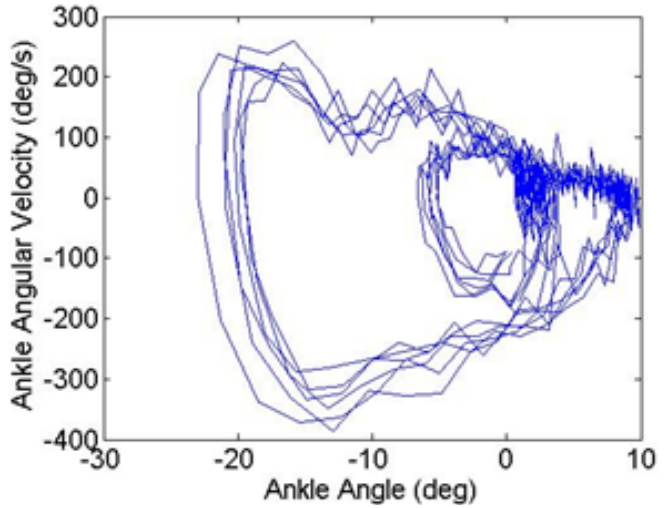

Figure 3 The phase plot of the ankle joint angle vs ankle angular velocity.

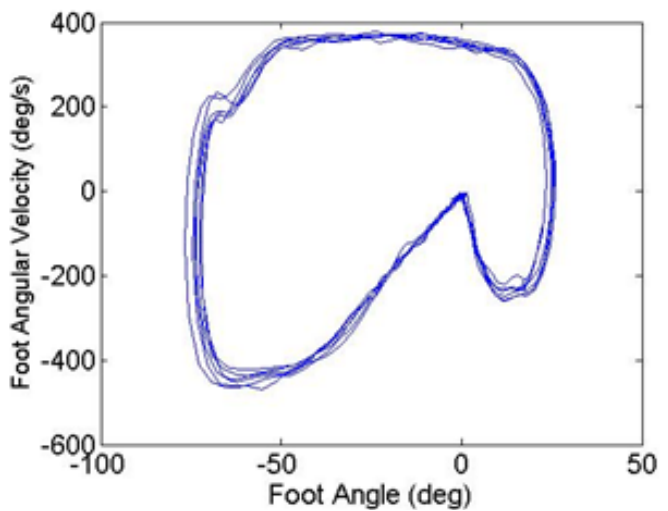

Figure 4 The phase plot of the foot elevation angle vs foot angular velocity.

The knee and shank angles for the same six cycles are shown in Figure 5. The shank elevation angle is smooth with one peak and minimum while the knee angle has two peaks and minima. Again, the elevation angle is smooth and repeatable.

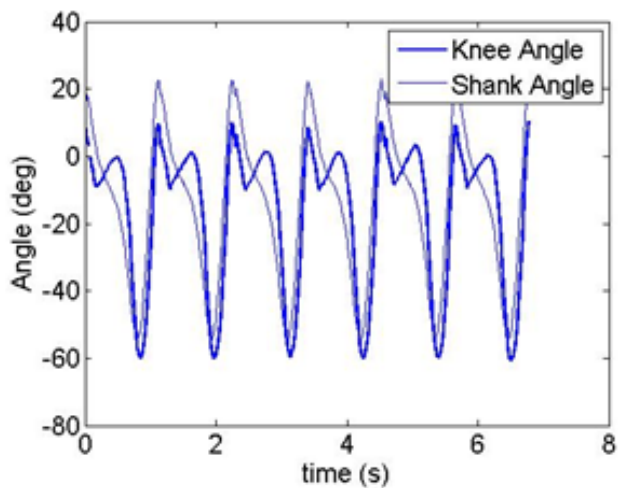

Figure 5 The knee joint angle and shank elevation angle are shown for six gait cycles.

The knee angle and shank angle were numerically differentiated to determine their respective angular velocities. A phase plot demonstrating the relationship between knee angular velocity and knee angle throughout the gait cycle is shown in Figure 6. Each of the six gait cycles shown in Figure 6 form two loops per gait cycle. The data appears noisy and is variable. 


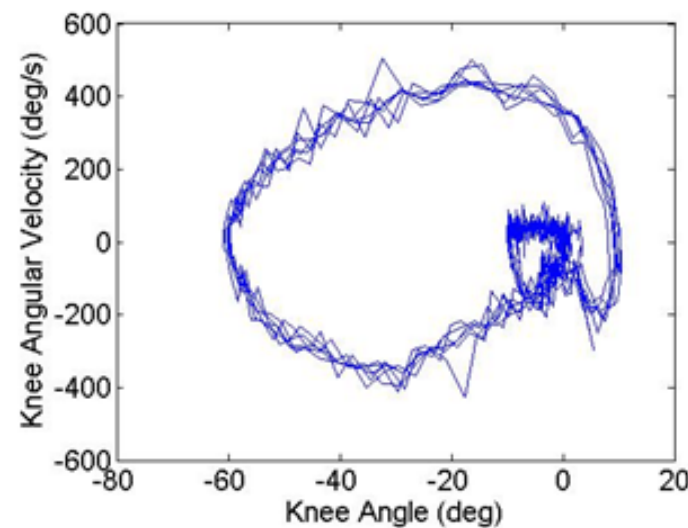

Figure 6 The phase plot of the knee joint angle vs the knee angular velocity.

Similar to Figure 4, a phase plot of shank angular velocity and the shank angle includes only one loop per gait cycle and the data is smooth with little variability. Because there is only one loop per cycle, each phase angle corresponds to a unique point in the gait cycle.

These findings are consistent with previous efforts using shank phase angle to determine percent gait cycle to control a powered prosthetic ankle. ${ }^{6}$ In the work by Holgate, shank phase angle was found to be consistent across varied stride lengths. ${ }^{6}$

The ankle phase angle calculated using data from Figure 3 is shown in Figure 7. It is determined by converting each point in the phase plot into polar coordinates. The distance to each point is measured by a length $\mathrm{r}$, and the angle is measured by Phi. The ankle phase angle data in Figure 7 is noisy because the data in Figure 3 is noisy.

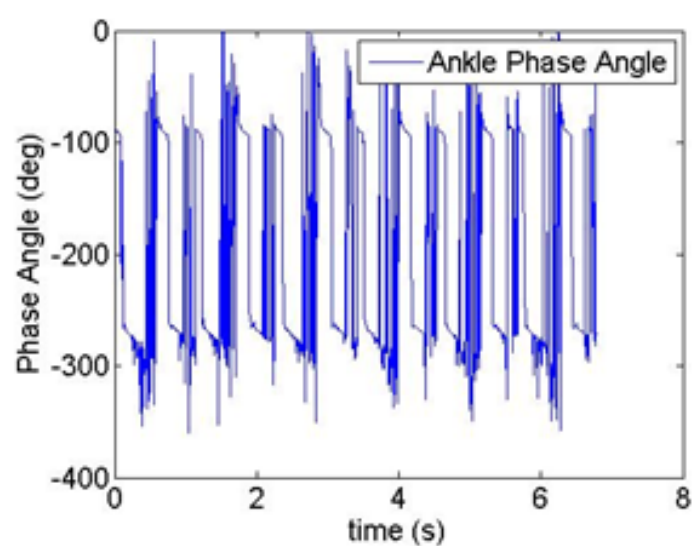

Figure 7 The ankle phase angle is shown for six gait cycles. The angle is unwrapped.

In Figures 3, 4, 6, \& 8, the scales of the vertical and horizontal axes are not the same. The range of angular velocities is very large as compared to the range of angles. Because of the paucity of data points between -50 and $50 \mathrm{deg} / \mathrm{s}$ in Figure 6, phase angles are not close to 0,180 , or 360 degrees in Figure 9. This same reasoning follows for Figure $3 \& 7$, Figure $4 \& 10$, and Figure $8 \& 11$. The phase points in the plots are connected by lines which can make it seem that there are more data points than are actually collected.

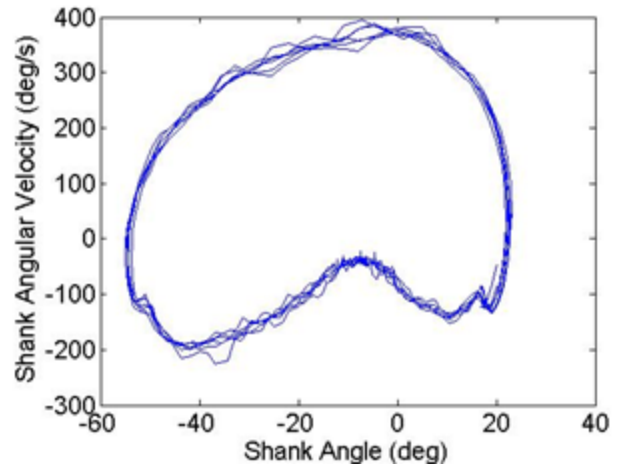

Figure $\mathbf{8}$ The phase plot of the shank elevation angle vs shank angular velocity.

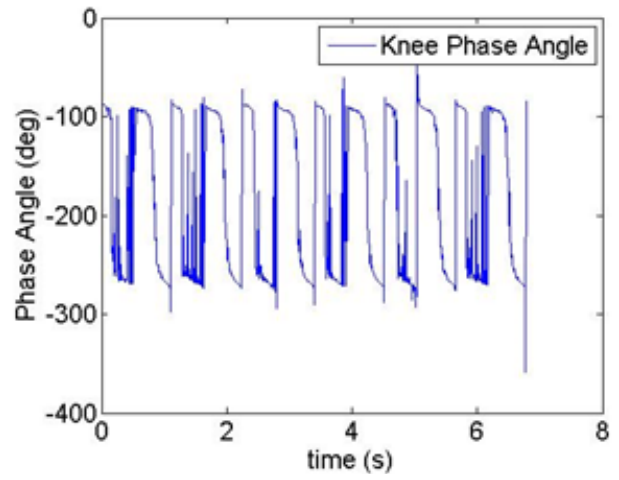

Figure 9 The knee phase angle is shown for six gait cycles. The angle is unwrapped.

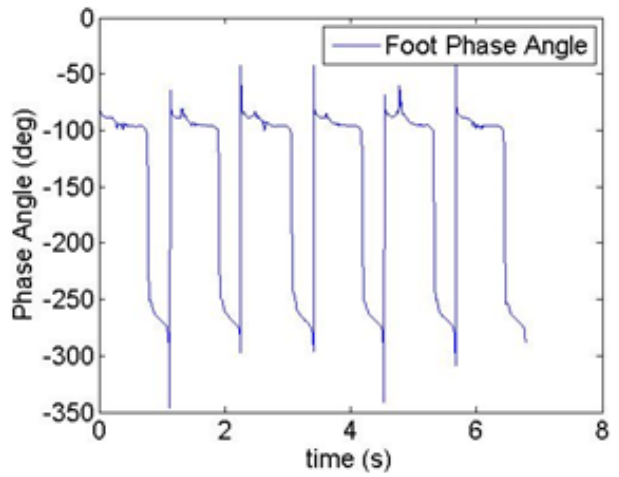

Figure 10 The foot phase angle is shown for six gait cycles. The angle is unwrapped.

The knee phase angle calculated using data from Figure 6 is shown in Figure 9. Because the data in Figure 6 is noisy, the knee phase angle is noisy.

Lastly, the phase angle of the knee was plotted against the phase angle of the ankle. The axes have equal length to determine if there is any relationship or signature pattern. The data is scattered in four areas, because both phase angles are noisy producing large jumps in phase angle data. All data is shown and no outliers are removed (Figure 12). 


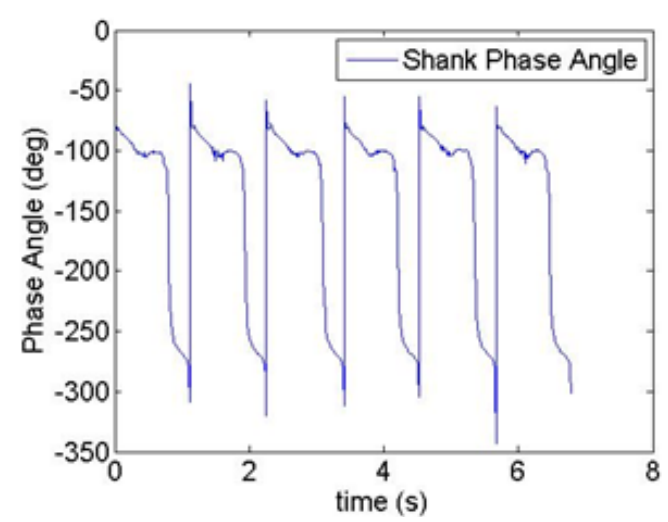

Figure II The shank phase angle is shown for six gait cycles. The angle is unwrapped.

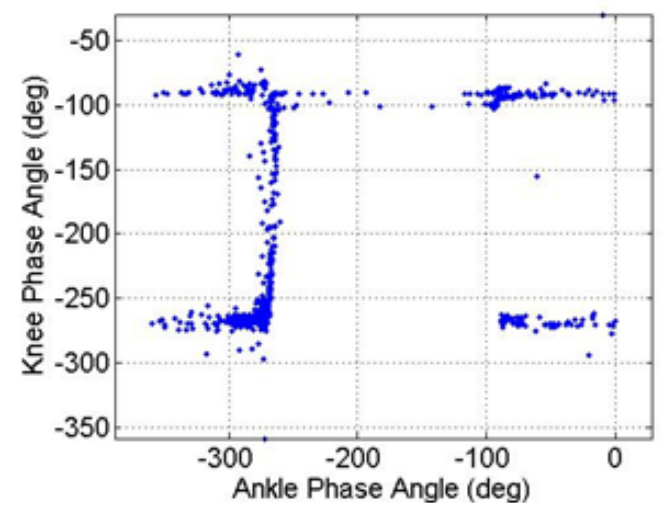

Figure I 2 The ankle phase angle vs the knee phase angles are shown for six gait cycles. The angles are unwrapped.

The foot phase angle calculated using data from Figure 4 is shown in Figure 10. The foot phase angle is smooth because the data in Figure 4 has little variability.

The shank phase angle calculated using data from Figure 8 is shown in Figure 11. The shank phase angle is smooth because the data in Figure 8 is smooth. Holgate $i^{6}$ uses the phase angle of the shank to act as a metronome to control his robot. He uses the polar length as a measure of stride length. If the stride length, percent gait cycle, and gait duration are known, then the velocity of the person can be determined.

The phase angle of the shank was plotted against the phase angle of the foot, see Figure 13. The axes have equal length to determine if there is any relationship or signature pattern. All data is shown and no outliers are removed. A signature pattern is shown relating the shank phase angle to the foot phase angle. First, the foot phase angle decreases quickly from -100 to -250 degrees while the shank phase angle changes very little. Then, the shank phase angle decreases quickly while the foot phase angle changes very little.

The same data analyses were repeated for six additional gait cycles from the fast walk condition. The pattern of the shank phase angle and foot phase angle is reported in Figure 14. The average gait cycle duration was $0.909 \mathrm{~s}$ which is $20 \%$ shorter than the previous data set. It should also be noted that in the fast walk condition stride length increased and cycle duration decreased. As the stride length, increases, the size of the loop in Figure 8 increases but the shape remains very similar. ${ }^{6}$

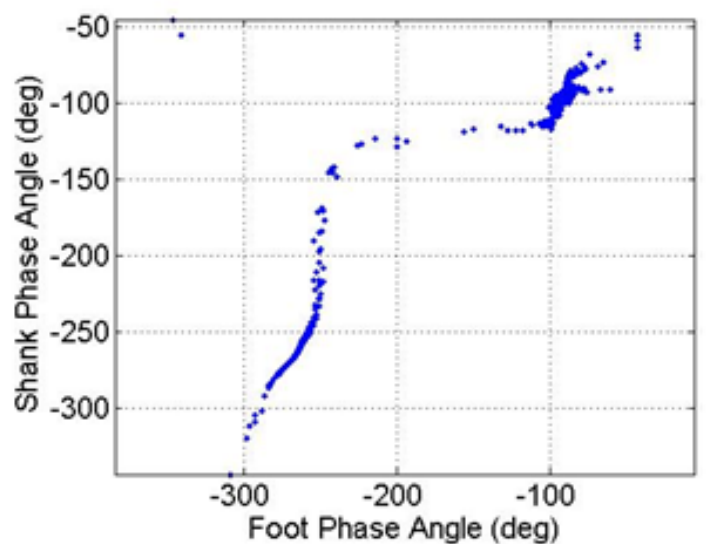

Figure 13 The foot phase angle vs the shank phase angles are shown for six gait cycles. The angles are unwrapped. (I.I42s duration for walking cycles).

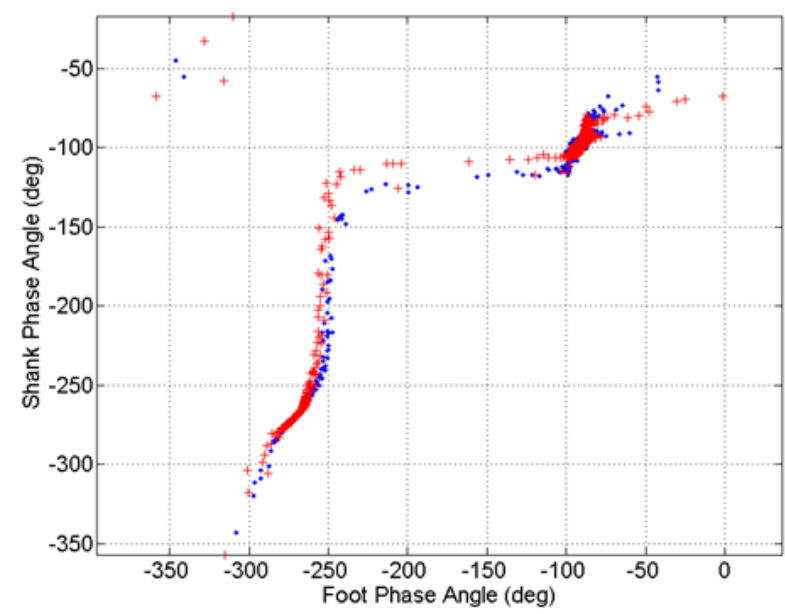

Figure 14 The foot phase angle vs the shank phase angles are shown for 12 gait cycles. The angles are unwrapped.The second group of gait cycles are for a person walking very fast, and are shown in red +'s (0.909s duration for fast gait cycles). The first set of six gait cycles from Figure 13 are shown in blue dots.

It was remarkable to see the signature pattern shown in red +'s for the fast walking gait cycles closely matches the pattern for moderate walking (Figure 13). A pattern in the ankle phase vs. knee phase was not detected.

The same data analyses were repeated for two gait cycles from the 10 degree (17.6\% grade) slope ascent and descent condition. A pattern was not detected in the ankle phase angle vs. the knee phase angle. The foot phase angle vs the shank phase angle, as shown in Figure 15, demonstrated a pattern similar to Figure 13.

In Figure 16, all data points for all trials and conditions are superimposed: walking, walking quickly, walking up a slope, and walking down a slope. The shank phase angle vs. the foot phase angle showed a consistent signature pattern for all conditions. There are slight differences between the curves, but they follow a similar pattern. 


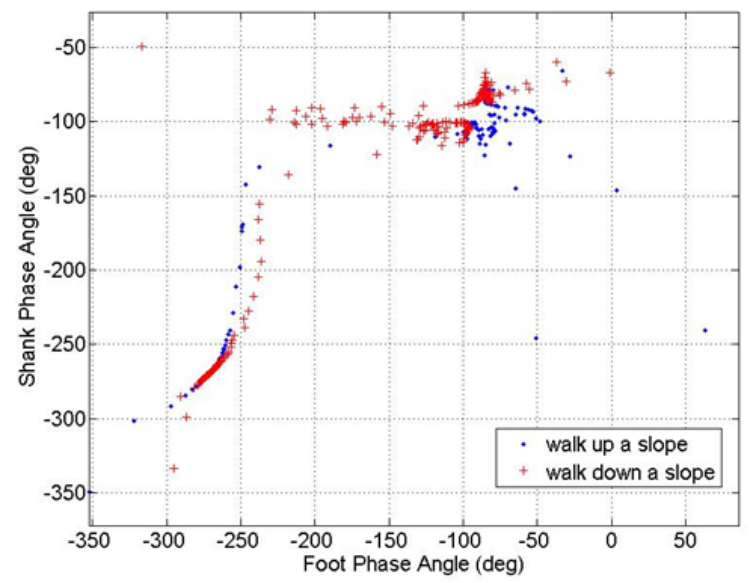

Figure I 5 The foot phase angle vs the shank phase angles are shown for 2 gait cycles. The angles are unwrapped. The first gait cycle is for walking up a 10 degree slope and the second gait cycle is for walking down a 10 degree slope (17.6\% grade)

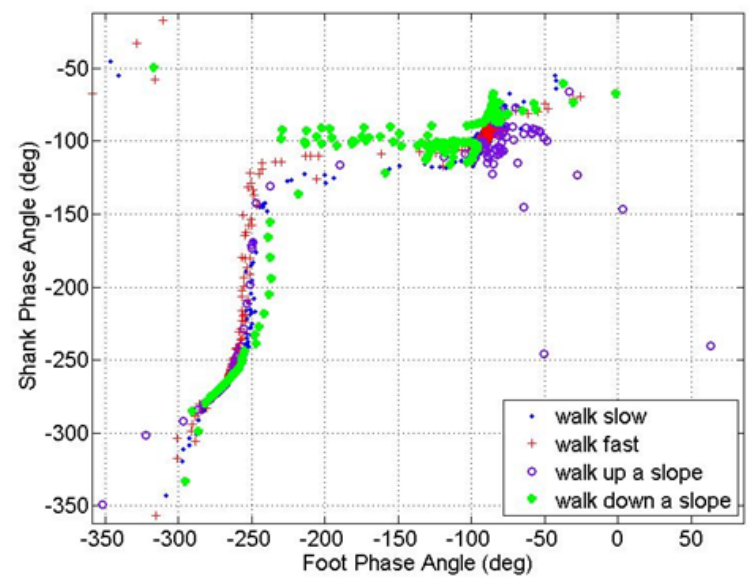

Figure 16 The foot phase angle vs the shank phase angles are shown for all data.

\section{Discussion}

Firstly, should one focus on or use elevation angles when controlling a biped or prosthetic ankle? We believe there are very important reasons to use elevation angles. Elevation angles are a measure of the moment caused by gravity against the limb. They are also a measure of whether the leg will strike or scuff the ground during gait. Lastly, the variability is low and they are very repeatable. Marken ${ }^{19}$ shows that the variables the human use to control a task are those variables with the least amount of variability even when the system has perturbations. In his example, if you want to determine which variable is being controlled by a heating system, you look for a variable that remains constant even if a window is opened. The temperature remains constant in a heating control system and is thus the "controlled variable." We argue that the human is controlling the elevation angles because they are very smooth and repeatable.

Secondly, it has been said elevation angles are not important because one must still convert back to joint angles, for example, to control the ankle joint. We disagree because a low level feedback loop does not have to be around a joint angle. For example, we use the progression of the shank phase angle to determine the percent gait cycle. Then, the percent is used to determine the amount of pull needed on a spring in our robotic tendon actuator. We have a low level feedback loop around the input or proximal position of the spring. We do not calculate joint angles when controlling our prosthetic ankle.

Lastly, the analysis of elevation angles is not new, but we believe Figure $13,14,15, \& 16$ are new. We show that the two phase angles progress together in a particular signature pattern regardless of the tasks of walking, walking quickly, and walking on slopes. In these tasks, the joint angles vary, and the phase loops might grow or shrink in size. For example, when walking fast with a larger stride length, the polar radius increases in size. Even though the polar radius changes, the progression of the two phase angles remains similar in the different tasks. We believe this is important because the progression of the shank phase angle can be used as a metronome in our robots. If the shank phase angle is determined using sensors, the foot phase angle can be calculated to determine the desired position and velocity of the foot. We use this data to control the force applied to the foot. In our previous work, we showed that the shank phase angle is invariant to different stride lengths. ${ }^{6}$

\section{Conclusion}

In the Human Machine Integration laboratory, we have been designing wearable robots. For our robotic systems, the user's intent must be determined to generate an appropriate motor output. We are developing controllers based on elevation angles because they are smooth and repeatable. We have been studying the phase angle of the shank and foot to determine their progression and to find any invariant patterns. We believe the signature pattern shown in Figure 16 is invariant

In our robotic systems, we use the shank phase angle as a metronome to determine the percent gait cycle of the user. In this work, we showed the shank and foot phase angles progress in a signature pattern for different walking speeds and walking up or down slopes. We believe this finding is unique and that the shank phase angle can be used to control bipeds and prostheses. In the future, we are focusing on studying the underlying patterns of the phase angles expanding our work to jogging and running.

\section{Funding}

The authors would like to acknowledge the grant that was awarded and administered by the U.S. Army Medical Research \& Materiel Command (USAMRMC), under Contract Number: W81XWH-0710193. The views, opinions, findings, information and presentations made do not necessarily reflect the position of the government and no official endorsement should be made. The opinions contained in this publication are those of the authors and do not necessarily reflect those of the funding agency.

\section{Acknowledgments}

This work was supported in part by the U.S. Department of Defense.

\section{Conflicts of interest}

The authors declare that there was no conflict of interest. 


\section{References}

1. Boehler AW, Hollander KW, Sugar TG, et al. Design, implementation and test results of a robust control method for a powered ankle foot orthosis (AFO). 2008 IEEE International Conference on Robotics and Automation; 2008 May 19-23; Pasadena, USA: IEEE; 2008.

2. Hollander KW, Ilg R, Sugar TG, et al. An efficient robotic tendon for gait assistance. J Biomech Eng. 2006;128:788-791.

3. Hitt JK, Bellman RD, Holgate MA, et al. The SPARKy (Spring Ankle with Regenerative Kinetics) Project: Design and Analysis of a Robotic Transtibial Prosthesis with Regenerative Kinetics. International Design Engineering Technical Conferences \& Computers and Information in Engineering Conference. 2009:1587-1596.

4. Holgate MA, Hitt JK, Bellman RD, et al. The SPARKy (Spring Ankle with Regenerative kinetics) project: Choosing a DC motor based actuation method. 2008 2nd IEEE RAS \& EMBS International Conference on Biomedical Robotics and Biomechatronics; 2008 Oct 19-22; Scottsdale, USA: IEEE; 2009.

5. Hollander KW, Sugar TG. A Robust Control Concept for Robotic Ankle Gait Assistance. 2007 IEEE 10th International Conference on Rehabilitation Robotics; 2007 June 13-15; Noordwijk, Netherlands: IEEE. 2008.

6. Holgate MA, Boehler AW, Sugar TG. Control algorithms for ankle robots: A reflection on the state-of-the-art and presentation of two novel algorithms. 2008 2nd IEEE RAS \& EMBS International Conference on Biomedical Robotics and Biomechatronics; 2008 Oct 19-22; Scottsdale, USA: IEEE; 2009.

7. Borghese NA, Bianchi L, Lacquaniti F. Kinematic determinants of human locomotion. J Physiol. 1996;494(Pt 3):863-879.

8. Grasso R, Bianchi L, Lacquaniti F. Motor patterns for human gait: backward versus forward locomotion. J Neurophysiol. 1998;80:18681885 .

9. Lacquaniti F, Grasso R, Zago M. Motor Patterns in Walking. News Physiol Sci. 1999;14:168-174.

10. Lacquaniti F, Ivanenko YP, Zago M. Kinematic control of walking. Arch Ital Biol. 2002;140:263-272.

11. Ivanenko YP, Grasso R, Macellari V, et al. Two-thirds power law in human locomotion: role of ground contact forces. Neuroreport. 2002;13:1171-1174.

12. Ivanenko YP, Grasso R, Macellari V, et al. Control of foot trajectory in human locomotion: role of ground contact forces in simulated reduced gravity. J Neurophysiol. 2002;87:3070-3089.

13. Hicheur H, Terekhov AV, Berthoz A. Intersegmental coordination during human locomotion: does planar covariation of elevation angles reflect central constraints?. J Neurophysiol. 2006;96:1406-1419.

14. Ivanenko YP, Cappellini G, Dominici N, et al. Modular control of limb movements during human locomotion. J Neurosci. 2007;11149-11161.

15. Ivanenko YP, d'Avella A, Poppele RE, et al. On the origin of planar covariation of elevation angles during human locomotion. $J$ Neurophysiol. 2008;99:1890-1898.

16. Sun H, Metaxas D. Animation of Human Locomotion Using Sagittal Elevation Angles. Proceedings the Eighth Pacific Conference on Computer Graphics and Applications; 2000 Oct 5-5; Hong Kong, China: IEEE; 2002.

17. Blaya JA, Herr H. Adaptive control of a variable-impedance ankle-foot orthosis to assist drop-foot gait. IEEE Trans Neural Syst Rehabil Eng. 2004;12(1):24-31

18. Blaya JA. Force-Controllable Ankle Foot Orthosis (AFO) to Assist Drop Foot Gait. Massachusetts Institute Of Technology. 2002;

19. Marken RS. Controlled Variables: Psychology as the Center Fielder Views It. The American Journal of Psychology. 2001;114:259-281. 